\title{
\%
}

Clara Crespo*

Marta Galiano**

Álvaro López-Barceló*

Alberto Merchante*

Agustín Navarro de Vicente-Gella*

Vicente J. Montes Gan*

\section{CONVERSACIONES SOBRE ECONOMÍA INTERNACIONAL: RESUMEN DE LOS PRINCIPALES ASPECTOS TRATADOS EN EL «WORKSHOP IN INTERNATIONAL ECONOMICS 2019»}

El objetivo de este artículo es situar al lector en la frontera del debate sobre economía internacional, a través de un resumen de los mensajes más destacados del «Workshop in International Economics 2019». Se presentaron diez ponencias de investigadores de primer nivel sobre cuestiones tan diversas como globalización, estrategias de educación de los hijos, medición de la inflación, mecanismos de decisión, expectativas, geografía, poder de mercado, distribución de la renta, rendición de cuentas del poder político o el papel de la ciencia económica. Las ponencias y el diálogo con los asistentes permiten extraer dos lecciones principales. La primera, que el debate en el ámbito de la economía internacional se orienta crecientemente a la intervención del Estado, frente a la corriente liberalizadora de años anteriores. La segunda, que las nuevas metodologías de tratamiento de datos ofrecen interesantes retos y oportunidades de análisis en áreas tan diversas como la medición de la inflación o el clima y la geografía física.

Palabras clave: economía internacional, globalización, educación, inflación, elección individual, expectativas, poder de mercado, política monetaria, rendición de cuentas.

Clasificación JEL: E64, F01, O15.

\section{Introducción}

La Asociación de Técnicos Comerciales y Economistas del Estado (ATCEE) organiza

\footnotetext{
${ }^{*}$ Miembros del Cuerpo Superior de Técnicos Comerciales y Economistas del Estado.

${ }^{\star *}$ Doctora en Economía.

Versión de noviembre de 2019.

DOI: https:/doi.org/10.32796/bice.2019.3118.6936
}

desde 2009 el «Workshop in International Economics» (WIE) en el Real Colegio Complutense en la Universidad de Harvard ${ }^{1}$. Este encuentro se articula en torno a una decena de sesiones intensivas sobre economía internacional lideradas por los profesores más destacados en $\triangleright$

\footnotetext{
1 En su edición del año 2019, el WIE tuvo lugar entre los días 16 y 19 de septiembre. Organizado por la ATCEE, su director académico es el profesor Diego Comín, del Dartmouth College, y está patrocinado por la Fundación Rafael del Pino y el ICEX España Exportación.
} 
cada una de las disciplinas analizadas, provenientes de las mejores universidades de Nueva Inglaterra, en especial, de la Universidad de Harvard.

En el año 2019, protagonizaron el WIE los siguientes profesores: Dani Rodrik, Fabrizio Zilibotti, Roberto Rigobon, David Laibson, David N. Weil, Andrei Shleifer, Susanto Basu, Diego Comín, Gerard Padró-i-Miquel y Esther Duflo. Estos autores presentaron tesis disruptivas que replantean numerosos modelos económicos tradicionales, fundamentalmente, en las áreas de la economía del comportamiento y del bienestar, el comercio internacional y la respuesta monetaria a las crisis económicas.

Si una de las dos lecciones que pueden extraerse del Workshop, en términos generales, ha sido que el péndulo del debate en el campo de la economía internacional se aleja progresivamente de la liberalización y se acerca, de nuevo, a la intervención del Estado; la otra lección ha sido la importancia y las enormes posibilidades que ofrece la conectividad global y que presentan hoy en día los sistemas de tratamiento de datos para fortalecer el análisis en este y en otros ámbitos de la ciencia económica.

A este respecto, el profesor de la Harvard Kennedy School, Dani Rodrik, argumentó que, en su opinión, la liberalización comercial ha ido demasiado lejos, ya que la compensación a los perdedores de la misma no ha sido evaluada correctamente y, en la mayoría de los casos, ha sido ínfima o inexistente, además de requerir una financiación vía impuestos que ha inducido, a su vez, una pérdida de eficiencia. Las tesis del profesor Rodrik se alinearon con las de la profesora del MIT (Instituto Tecnológico de Massachusetts) y Premio Nobel de Economía 2019, Esther Duflo, que afirmó que, en general, los costes de ajuste reales en este campo son muy superiores a los estimados tradicionalmente por los economistas. $Y$ ello porque, más allá de la mejora económica, hay otros aspectos que las personas consideran prioritarios, como la dignidad, objetivos vitales, el reconocimiento social, etcétera. En este sentido, el profesor de la Universidad de Yale, Gerard Padró-i-Miquel, presentó un modelo de ciencia política con el que trató de explicar la falta de accountability de los dirigentes en las sociedades fragmentadas o heterogéneas.

Estas «debilidades» analíticas que, de acuerdo con estos autores, caracterizan el trabajo de muchos estudiosos de la economía, contrastan con el potencial de las nuevas metodologías e instrumentos para el tratamiento de datos. En este sentido, Roberto Rigobon, profesor en MIT Sloan, describió sus sistemas alternativos para la medición diaria de la inflación en una amplísima lista de países del mundo y, David Weil, profesor de Brown University, disertó sobre el clima y la geografía física, a partir de sus estudios más recientes, en los que hace un uso intensivo del tratamiento de datos para concluir que la localización de la población está influida por la geografía.

Tanto Susanto Basu como Diego Comín, profesores del Boston College y del Dartmouth College, respectivamente, analizaron por qué la participación de las rentas del trabajo en la renta nacional se ha reducido durante las últimas décadas en los países desarrollados. Tras considerar el shock tecnológico y la globalización como variables explicativas, el primero describió el impacto del poder de mercado de las empresas en el mercado de trabajo, mientras que el segundo desagregó la participación de las rentas del trabajo observando que la masa salarial ha crecido en los trabajos de baja y alta cualificación, y se ha deprimido en los de media.

Se analizó, además, la necesidad de abordar la problemática económica actual desde $\triangleright$ 
un punto de vista interdisciplinar, incluyendo nuevas políticas, como los nudges, o empujones, defendidos por el profesor de la Universidad de Harvard, David Laibson, o el paternalismo de Fabrizio Zilibotti, profesor de la Universidad de Yale.

Finalmente, Andrei Shleifer, de la Universidad de Harvard, presentó sus trabajos sobre expectativas que revolucionaron la teoría económica al demostrar que las expectativas no son racionales, sino que presentan errores sistemáticos que tienen un impacto en las variables reales.

A continuación se resumen las principales conclusiones extraídas por los participantes en el WIE 2019 de las ponencias desarrolladas por estos autores.

\section{Conversaciones con expertos en economía internacional}

\subsection{Globalización, mercado de trabajo y populismo. Dani Rodrik, Harvard Kennedy School of Government}

Como se ha señalado, el profesor de la Universidad de Harvard, Dani Rodrik, puso de manifiesto que, en su opinión, la liberalización comercial podía haber ido demasiado lejos (Rodrik, 1997 y 2011). Partiendo del análisis Heckscher-Ohlin de libre comercio clásico y del teorema de Stolper-Samuelson, Rodrik elabora un índice de la ratio coste-beneficio político de la liberalización comercial. Concluye que, en la práctica, la necesaria compensación de los perdedores de la liberalización comercial es ínfima o no llega; esta requiere financiación mediante impuestos, que a su vez generan una pérdida de eficiencia; y la compensación necesaria en los casos de shallow integration, es decir, la reducción de un arancel que ya era bajo, es mayor en valor absoluto que la ganancia de eficiencia que conlleva.

El conocido artículo de Autor et al. (2016) sobre el China shock, una expresión que utilizaron varios ponentes del WIE, apunta a un gran impacto distributivo en los salarios y el empleo debido a la competencia china. Como corolario, a medida que el capital es más móvil, la carga impositiva recae más sobre el trabajo.

Si lo que se plantea es una integración más profunda (armonización regulatoria, normas de protección de la propiedad intelectual, etcétera), no está claro que se generen ganancias de eficiencia. Además, la regulación, como manifestación de los juicios de valor de una sociedad, es un resultado del contrato social propio de cada país. Cuando dos países comercian, se crea la posibilidad de arbitraje entre ambos sistemas regulatorios. Las encuestas de opinión revelan que los ciudadanos perciben que el comercio genera una discrepancia entre las reglas de iure y las de facto ${ }^{2}$.

Finalmente, se debatió sobre si, para cada país, en cada momento del tiempo, existe un punto óptimo de globalización, que sería aquel en el que las ganancias en términos de eficiencia fueran superiores a los aumentos de desigualdad que genera (Lang y Tavares, 2018).

Rodrik se pregunta en sus trabajos por las implicaciones de esta cuestión para las elecciones políticas: la percepción de inseguridad económica considera que genera populismo, tanto de derechas como de izquierdas. Señaló que el mejor ejemplo de populismo de izquierdas, a su modo de ver, fue el New Deal de Roosevelt. En los años treinta en EE UU, la ruptura del patrón oro y del resto de $\square$

Clásico ejemplo de producción textil en un país desarrollado o en uno en desarrollo, con estándares laborales diferentes. 
Crespo, Galiano, López-Barceló, Merchante, Navarro de Vicente-Gella y Montes Gan

propuestas de corte keynesiano pueden considerarse medidas de izquierdas pensadas para reenganchar socialmente a una población descontenta e insegura que viraba hacia el populismo de derechas. En los países donde se produce este tipo de descontento, ¿deben los Gobiernos aprobar medidas que hoy día se consideran populistas de izquierdas?

\subsection{La economía de las estrategias de paternidad. Fabrizio Zilibotti, Yale University}

Desde las aportaciones seminales de Gary Becker (1981), la ciencia económica ha prestado una creciente atención a los incentivos que subyacen en decisiones del ámbito familiar, como el número de hijos o la inversión en educación, entre otras. A esta rama de la literatura se han venido a sumar las contribuciones de Fabrizio Zilibotti y sus coautores en una serie de artículos (Doepke y Zilibotti, 2017; Doepke, Sorrenti y Zilibotti, 2019) y un libro (Doepke y Zilibotti, 2019) de reciente publicación, en los que se analiza la relación entre el contexto económico y la forma de educar a los hijos en el ámbito familiar.

Algunos trabajos anteriores ya habían abordado la cuestión de "cuánto» invertir en educación, bien en el hogar o fuera de él. La novedad consiste en adentrarse en la - hasta ahoracaja negra del "cómo» se educa a los hijos en la familia y si hay variables económicas que condicionen la elección de los padres, de una forma u otra, de interactuar con sus hijos.

Para ello, estos autores parten de la clasificación de Baumrind (1967), clásica en la psicología del desarrollo, que distingue tres estilos de paternidad: (i) permisivo, dejando a los hijos tomar sus propias decisiones; (ii) autoritativo, estableciendo ciertas normas que se razonan con los hijos de modo que se van moldeando sus propias preferencias; y (iii) autoritario, que supone un control estricto sobre los hijos y la toma de decisiones exclusivamente por parte de los padres.

A partir de ahí, se establece un modelo con supuestos comunes en economía, pero introduciendo algunas variables novedosas. Los padres son agentes racionales que maximizan su utilidad intertemporal, que derivan del consumo, tiempo de ocio y bienestar de los hijos, movidos en este último caso por sentimientos altruistas y paternalistas. A su vez, los padres se enfrentan a las restricciones presupuestaria $y$ temporal, $y$ deciden sobre el nivel de autonomía de los hijos en sus elecciones, sabiendo que sus preferencias no son necesariamente coincidentes en relación con el tiempo dedicado al ocio y la adquisición de habilidades cognitivas y no cognitivas.

En este contexto, la elección de un estilo de paternidad permisivo, autoritativo o autoritario vendrá determinada por el retorno que se obtiene del esfuerzo dedicado a fomentar la adquisición de habilidades en los hijos. Así, cuando la desigualdad económica es limitada y el retorno de la inversión en capital humano se diluye, los padres tendrán menos incentivos a optar por una estrategia de paternidad autoritaria o autoritativa, también caricaturizada a través de la figura de las «madres tigre» o los «padres helicóptero».

Las predicciones de este modelo son consistentes con lo observado en diferentes países y a lo largo del tiempo, utilizando varios proxies de las distintas estrategias de paternidad, tales como el número de horas que los padres dedican a los hijos, o la importancia que se atribuye en encuestas a la transmisión de determinados valores como la obediencia, el $D$ 
trabajo duro, la imaginación o el pensar por uno mismo.

En aquellos países donde existe mayor desigualdad económica y, por tanto, mayor retorno de la educación, se observa cómo los padres se decantan por estrategias de paternidad más intensivas (autoritarias o autoritativas), frente a sociedades más igualitarias donde adquiere mayor presencia el estilo permisivo. Del mismo modo, se aprecia una creciente predominancia de la paternidad intensiva desde los años ochenta y hasta hoy, coincidiendo en el tiempo con la creciente desigualdad y el mayor retorno relativo del esfuerzo en educación. Mientras que en épocas con menores niveles de desigualdad, como fue el caso entre la segunda guerra mundial y los años setenta, se popularizó la idea de una paternidad más permisiva.

\subsection{El misterio de la inflación oculta, Roberto Rigobon, MIT Sloan}

Durante la última década, hemos visto cómo las políticas monetarias expansivas de gran alcance de los bancos centrales, como la Large-Scale Asset Purchase de la FED o el Quantitative easing del BCE, no han provocado repuntes en la inflación. Igualmente, en los últimos años ha tenido lugar un aumento de la demanda agregada en Europa y EE UU, acompañado de un aumento de las tensiones proteccionistas que tampoco ha dado lugar a un aumento en la inflación.

Esta ausencia de inflación, contraria a toda teoría económica, es lo que lleva al profesor Rigobon a plantearse si existe una inflación oculta similar, de alguna forma, a la dark matter postulada por Hausmann y Sturzenegger (2006) para explicar la diferencia entre las estadísticas estadounidenses de la cuenta corriente acumulada de EE UU y las estimaciones basadas en el rendimiento real de posición de inversión internacional neta.

Rigobon señala que las circunstancias descritas anteriormente sí que han provocado inflación, lo que pasa es que se trata de una inflación oculta por una serie de razones, y atribuye este anómalo comportamiento de los precios a dos causas. En primer lugar, es posible que los institutos de estadística nacionales estén midiendo mal la inflación al emplear técnicas que bajo algunos supuestos son, como veremos, poco representativas. En segundo lugar, el auge del comercio electrónico y del comercio de datos hace que las mediciones de inflación y PIB hayan quedado obsoletas para estos sectores por no incluirse el pago con datos del consumidor que suele acompañar a numerosas transacciones electrónicas.

A continuación analizaremos las dos formas más representativas de esta distorsión: el quantum pricing, i.e., una segmentación abrupta de los precios por ciertas empresas, y el data payment, la idea de que las plataformas online pueden incurrir en pequeñas pérdidas en la provisión de bienes y servicios si a raíz de la transacción reciben una cantidad de datos suficientemente valiosa, lo cual es una parte del precio que no se incluye en la contabilidad nacional por escapar a la definición tradicional de inflación o de PIB.

Acerca de la nueva forma de medir la inflación, Rigobon defiende que medir la evolución de los precios al consumo mediante una cesta que se asume representativa es una forma ineficiente de medir la inflación en el siglo XXI. En la actualidad, la práctica totalidad de los comerciantes publica, a través de su tienda online, en tiempo real, los precios de los múltiples productos que oferta, por lo que, haciendo un seguimiento automatizado de todos estos $\triangleright$ 
precios, se puede hacer un cálculo de la inflación que sea en tiempo real, a la vez que conste de una base de productos infinitamente mayor a la que pueda recopilar un grupo humano de encuestadores.

Esto es exactamente lo que desarrolla su proyecto $^{3}$, en el que utiliza los precios de todos los productos de las páginas de comercio electrónico en una amplia lista de países para medir su inflación diaria. Es interesante notar que, en el caso de España, los puntos mensuales del INE (Instituto Nacional de Estadística) coinciden con la inflación medida por Rigobon, pero las diferencias intramensuales son sustanciales. En un futuro, aunando la información ya disponible con datos sobre los volúmenes de transacciones se podría calcular la inflación usando no los precios ofrecidos por los vendedores, como se hace actualmente, sino los precios a los que tienen lugar las transacciones, lo cual puede ser relevante en mercados poco líquidos.

También es destacable el bajo coste de esta técnica, que en principio no usa encuestadores a pie de calle, sino algoritmos automatizados de búsqueda y seguimiento de precios online: con un presupuesto de poco más de 5 millones de dólares anuales, Rigobon es capaz de medir, en tiempo real y con extraordinaria precisión, la inflación de un amplio grupo de países, entre ellos España. Por motivos de altruismo, estos datos se comparten gratuitamente con los bancos centrales de los países involucrados, que obtienen así una «segunda opinión» sobre la inflación medida por los servicios de estadística nacionales.

Por otra parte, Roberto Rigobon profundizó en dos estrategias de precios del comercio minorista por internet: quantum pricing y data payment.

3 The Billion Prices Project, http://www.thebillionpricesproject.com
1. Quantum pricing. La inmensa mayoría de tiendas de textiles y comercios online adoptan una política de fijación de precios consistente en ofertar un número dado de precios $(19,99 ; 29,99 ; 39,99$ y pocos más). Inditex, Uniqlo y empresas de este mercado etiquetan por «tipos de precios", y los cambios en los precios discurren de manera no lineal. Mantienen sus productos en un tipo de precio acumulando pérdida de valor real hasta que los cambian de tipo. No siempre saltan al escalón superior, sino que algunos bajan al anterior también. Por tanto, en conjunto, en la cesta de la tienda se puede observar inflación, pero no se puede observar una trayectoria de inflación siguiendo solo algunos productos. Si la cesta usada para calcular la inflación se compone de productos sujetos al quantum pricing, no se observarán diferencias en la inflación calculada a largo plazo, pero sí unos escalones «efecto estadístico» en el corto. La forma de evitar tales errores de medición es, por consiguiente, computar la inflación no en base a una cesta, sino incluyendo todos los bienes ofrecidos por las empresas, tal y como hace The Billion Prices Project.

2. Data payment. Un gran número de servicios de la economía digital son «gratuitos» para sus usuarios: las búsquedas de Google, las redes sociales, los correos electrónicos, etcétera. Realmente, estos productos se «pagan» mediante el consumo de publicidad y el suministro de datos por parte de los usuarios, pero al no tratarse de transacciones monetarias, no entran en la definición de PIB ni son mensurables a efectos de la inflación. $D$ 
Tradicionalmente, la información se había organizado geográficamente y por grupo social, pero hoy día se puede organizar por preferencias individuales, que permiten predecir los intereses y compras de los potenciales consumidores. Cada producto vendido aporta información relevante para reorganizar los datos del resto de consumidores, por lo que para una plataforma como Amazon conviene bajar el precio del producto para atraer al último consumidor: la información que da esa compra genera más valor (en recomendaciones para futuras compras o en venta de espacios de publicidad) que la pérdida en la que se incurra por la venta del producto. El exponencial auge de este tipo de servicios hace que el volumen de servicios «pagados» mediante el suministro de datos sea mucho mayor que los aumentos en precios nominales, por lo que no siempre se puede medir. De ahí la contención de precios que se vive en los países desarrollados. Además, es posible que, al no imputar rentas a la utilización de los servicios gratuitos, estemos midiendo peor el PIB, y de ahí también el aplanamiento aparente del crecimiento de la productividad total de los factores.

Este modo de negocio ha superado recientemente las barreras de la nube, y ya es posible encontrar negocios físicos, como, por ejemplo, la cafetería Shiru Cafe del campus de la Universidad de Brown, donde el café es gratuito para los alumnos, que «pagan» el bien con sus datos y con consumo publicitario, por ejemplo, por una empresa interesada en dar a conocer a los estudiantes sus programas de graduate recruiting.

En conclusión, los cambios vividos en la última década a raíz del auge de comercio y prestación de servicios electrónicos hacen necesario replantearse la forma en la que medimos actualmente la inflación.

\subsection{Elección individual. David Laibson, Harvard University}

David Laibson, profesor de la Universidad de Harvard, disertó en un tono prointervención del Estado. A partir de varios experimentos, Laibson define una tasa de descuento adicional a la habitual, que llama sesgo presente y que implica que el individuo valore lo que sucede «aquí y ahora» el doble de lo que sucede en cualquier momento futuro. El resultado de este descuento hiperbólico es arrastrar los pies, o procrastination. Los ejemplos son innumerables: el individuo declara que va a dejar de fumar, solo que hoy no, que va a hacer dieta, ir al gimnasio, ahorrar para su vejez, etcétera, solo que empezará mañana. Por tanto, hay un argumento de bienestar público que habilita al Estado para intervenir estableciendo una norma que obligue a que el resultado final se acerque al deseo declarado, pero no cumplido, por el individuo.

Bajo el título Nudges are not enough. The case for hard paternalism, profundiza en la elección individual: por ejemplo, esquemas como ofrecer posibilidad de opt-in en un fondo de pensiones reciben menos inversión que aquellos en los que la situación por defecto es contribuir al fondo de pensiones y se permite el opt-out.

Para Laibson, el bienestar social mejora con la intervención normativa pública a pesar de la ligera reducción del bienestar de los individuos planificadores o que no tienen sesgo presente. El problema del siglo XIX, señala, era la pobreza en la vejez, hasta que el Estado obligó a ahorrar. Aplaude los sistemas europeos de pensiones por su participación obligatoria, aunque no por su financiación. Para el profesor, el problema del siglo XXI es la pobreza infantil: ¿qué intervención pública nos $\triangleright$ 
Crespo, Galiano, López-Barceló, Merchante, Navarro de Vicente-Gella y Montes Gan

proponemos? si son solo vales de comedor —nudges o incentivos-, no será suficiente.

\subsection{Geografía, desarrollo económico y población. David N. Weil, Brown University}

El profesor Weil también hace uso intensivo del tratamiento de datos de clima y geografía física, así como de la iluminación que se ve desde el espacio, para concluir que la localización de la población está influida por la geografía - terrenos fértiles, cercanía a las costas, etcétera-, especialmente en los países que se urbanizaron hace más tiempo. También establece una medida de sobrepoblación relativa con respecto a lo que predecirían las variables geográficas.

El resultado para España es que está relativamente infrapoblada de manera ligera, y que las poblaciones importantes deberían estar en la costa: la localización de Madrid es una anomalía histórica. Concluye también que la mayoría de los países africanos están relativamente sobrepoblados respecto a sus características físicas: cuanto más tarde en la historia sucede la transición demográfica, más rápido crece la población durante el periodo de transición.

El trabajo no está exento de críticas, pero se puede pensar que tiene una aplicación interesante: predecir los movimientos migratorios desde los países relativamente sobrepoblados en los que empeoran las variables físicas como consecuencia del cambio climático.

\subsection{Expectativas y crisis financieras. Andrei Shleifer, Harvard University}

La tesis medular del profesor Shleifer es afirmar que las expectativas de los agentes racionales contienen unos errores sistemáticos que inciden directamente en las variables reales, afirmación que sustenta usando como ejemplos los planes de inversión, los pánicos bancarios y, finalmente, la intervención de los banqueros centrales en la pasada crisis financiera. Por tanto, las predicciones plasmadas en encuestas sobre expectativas de los agentes racionales pueden ser comparadas con los valores posteriormente verificados para así medir el grado de racionalidad de los agentes encuestados.

En primer lugar, Shleifer recuerda la idea de Prescott de que las encuestas son un proxy relativamente débil para medir las expectativas, lo que esencialmente viene a significar que las expectativas en macro, al igual que la utilidad en micro, son esencialmente no observables, llegando así a la conclusión de que no podemos afirmar que las encuestas sobre expectativas sean predictores insesgados de la conducta de los agentes.

En el caso de los planes de inversión, Shleifer defiende que siguen con retardo las expectativas sobre rendimientos recabadas en encuestas. En concreto, defiende que las expectativas sobre rendimientos de las acciones son muy similares entre distintos grupos de agentes con distintos grados de información financiera y distinta vocación inversora, a la vez que están altamente correlacionadas con los anteriores rendimientos de la cartera.

Al no hallar una correlación positiva entre las expectativas de rendimientos y las predicciones de varios modelos de rentabilidad esperada, Shleifer sugiere que explicar la inversión con expectativas puede ser bastante más preciso que con la teoría tradicional, coincidiendo así con los resultados de Greenwood y Hanson (2013) sobre el mercado de bonos: altas primas de riesgo predicen rentabilidades excesivas en bonos corporativos. En síntesis, los $D$ 
errores de predicción parecen ser sistemáticos y recurrentes, observándose tanto un excesivo optimismo en tiempos de bonanza como un excesivo pesimismo en tiempos de crisis.

Posteriormente, Shleifer desarrolló tales errores de predicción respondiendo algunas de las preguntas planteadas en su reciente libro A crisis of beliefs (Gennaioli y Shleifer, 2019), sobre cuáles son los mecanismos de propagación de las crisis financieras, si pueden ser anticipadas, y si puede la política monetaria hacer algo para remediarlas. En concreto, hace notar que la burbuja inmobiliaria y la posterior crisis financiera de 2008 son esencialmente el típico auge de créditos seguido de una crisis y recesión, y que —esta es la contribución crucial- tanto los mercados como las autoridades monetarias no anticiparon la burbuja, pese al perfil similar en términos de expectativas dado por el entusiasmo en el mercado inmobiliario y las MBS (mortgage-backed security) que la alimentaron.

Para cuantificar en términos numéricos lo expuesto anteriormente, Shleifer toma una muestra de 34 países entre 1950 y 2016 y observa cómo, después de tres años de crecimiento en el precio de las acciones y de la deuda de las empresas, la probabilidad de crisis financiera en los siguientes tres años es del $45 \%$. Tal probabilidad alcanza «solo» el $39 \%$ si el crecimiento se ha registrado en el precio de las viviendas y la deuda de los hogares. En resumen, la euforia en los mercados de crédito suele ir seguida por un menor crecimiento económico. Las crisis se deben a creencias irracionales, que pueden, no obstante, verse amplificadas por los mecanismos tradicionales.

Finalmente, Shleifer disertó también sobre la crisis financiera de 2008 y sobre la decisión de las autoridades estadounidenses de no rescatar a Lehman Brothers, considerando que fue una mezcla de desconocimiento sobre el riesgo sistémico de Lehman y de querer establecer un precedente para evitar el riesgo moral. Respecto a la crisis de la zona euro de 2012, considera que la declaración de Draghi de salvar el euro «whatever it takes» cambió el curso de la historia al modificar abruptamente la tendencia de las expectativas, hasta ese momento cada vez más pesimistas.

\subsection{Poder de mercado y política monetaria. Susanto Basu, Boston College}

En primer lugar, el profesor Basu intenta responder a la pregunta de si deberían los bancos centrales fijar un objetivo no solo en términos de precios de consumo, sino también de precios de inversión, teniendo en cuenta que, desde los noventa, la mayoría de los bancos centrales han venido adoptando un objetivo de inflation targeting que considera únicamente la evolución de los precios al consumo, tales como el HICP (Harmonized Index of Consumer Prices) del BCE, que, pese a incluir bienes de consumo duradero, excluye los precios de los activos.

Tras mostrar que en EE UU la inflación medida mediante el deflactor PCE (Personal Consumption Expenditure) no correlaciona con el deflactor de la inversión no residencial, el conferenciante defiende que monitorizar también los precios de los activos es necesario porque los precios tanto de los bienes de consumo como de los de inversión son rígidos, y los shocks sectoriales están imperfectamente correlacionados.

Posteriormente, Basu presenta un modelo $2 \times 2$ neokeynesiano, con costes de ajuste y shocks tecnológicos sectoriales en el que $D$ 
demuestra que una regla tipo Taylor da un mejor resultado en términos de bienestar si no establece como meta el deflactor en un sector, sino en ambos, bienes de consumo y activos.

La segunda parte de la presentación consistió en desarrollar un modelo de función de costes para empresas con poder de mercado que permita contrastar, para el mercado estadounidense, la hipótesis de que el poder de mercado está aumentando.

Comparando datos sobre la participación del factor trabajo en la producción nacional de EE UU, es posible estimar el poder de mercado de las empresas vía sus tasas de beneficio (Basu, 2019) y llegar a la conclusión de que altas cotas de poder de mercado son incompatibles con la evidencia empírica observada en el mercado de trabajo, pues el poder de mercado ha aumentado como mucho un $10 \%$ desde 1980.

En conclusión, Basu defiende que un mayor poder de mercado debería haber supuesto menores niveles de empleo, mayor inflación y mayor crecimiento de la productividad total de los factores; al no verificarse ninguno de los tres resultados para el caso de EE UU, se puede concluir que o bien el poder de mercado no ha aumentado significativamente, o bien otros shocks positivos han compensado fortuitamente sus efectos macro.

\subsection{Polarización del mercado de trabajo. Diego Comín, Dartmouth College}

Diego Comín desagrega la participación de las rentas del trabajo observando que la masa salarial ha crecido en los puestos de trabajo de baja y alta cualificación, mientras que se ha deprimido en los de media cualificación. Tanto el empleo como los salarios se han polarizado.
El profesor Comín considera que, frente a las explicaciones tradicionales, este hecho se debe a la elevada elasticidad-renta de la demanda de servicios intensivos en trabajo con elevada cualificación, como la educación, la sanidad y los servicios profesionales, y con baja cualificación, por ejemplo, cuidados personales, respecto a los trabajos relativamente intensivos en cualificaciones medias, como la producción manufacturera.

A medida que la renta aumenta, cambia la composición del consumo y, con ello, la retribución de los trabajadores de cada sector. Utilizando tablas input-output para comprobar la hipótesis, Comín concluye que no hay «culpables» del aumento de la desigualdad.

\subsection{Rendición de cuentas en sociedades divididas. Gerard Padró-i-Miquel, Yale University}

El profesor de la Universidad de Yale, Gerard Padró i Miquel, expuso el modelo que ha desarrollado para explicar por qué los dirigentes poco democráticos del África poscolonial se mantenían en el poder durante largos periodos a pesar de la evidencia de su enriquecimiento personal y la ineficiencia de sus políticas, todo ello en perjuicio de sus ciudadanos. El supuesto de partida es que estos dirigentes se mantienen en el poder gracias al apoyo de los miembros de su comunidad o grupo étnico, y lo que se pretende es explicar por qué este grupo mantiene en el poder al líder a pesar de su notoria corrupción (Padró i Miquel, 2007).

Recordó que algunos autores han investigado en el pasado cómo la heterogeneidad de la población, ya sea étnica, lingüística o religiosa, por ejemplo, afecta negativamente al crecimiento económico y a la calidad de las políticas (Easterly y Levine, 1997). 
Padró i Miquel desarrolla un modelo que se fija en la rendición de cuentas del Gobierno cuando la población está dividida, con el fin de estudiar cómo la heterogeneidad puede afectar a los resultados económicos y políticos de un país aun en ausencia de un conflicto armado.

Se supone que hay dos grupos étnicos: $A$ y $B$. La proporción de individuos del grupo $A$ sobre la población total es $\Pi^{A}$. Suponemos que los individuos no pueden cambiar de grupo y que se puede identificar fácilmente el grupo al que pertenecen.

Al mismo tiempo, hay dos actividades económicas, a y $b$. Cada grupo tiene ventaja comparativa en una de las dos (puede ser por factores tales como localización geográfica, existencia de redes o aptitudes concretas). Por ello, aunque pueden cambiar de actividad, hacerlo supone reducir el rendimiento que obtienen de su actividad económica. Si un ciudadano del grupo $A$ obtiene, con el ejercicio de la actividad $a$, un rendimiento de $\omega^{a}$, ejercer la actividad $b$ le supondría un rendimiento equivalente a $\omega^{\mathrm{a}}-\theta^{A}$.

El Estado puede gravar las actividades económicas con impuestos $\tau^{a}$ y $\tau^{b}$ y puede emplear la recaudación para proporcionar gasto que favorezca a alguno de los dos grupos $\eta^{A}$ y $\eta^{B}$. Por ejemplo, una forma de discriminación en el gasto público es contratar solo a individuos de uno de los grupos para ocupar los puestos de trabajo de la Administración pública.

En cualquier momento dado, uno de los dos grupos étnicos está en el poder, con un líder $L^{i}$, donde $i$ denota el grupo étnico al que pertenece el líder, que estará apoyado por una estrecha élite de su mismo grupo. En ningún momento existiría ningún problema para encontrar candidatos a líder en ninguno de los dos grupos.
Cuando el líder recibe el apoyo de su grupo étnico, la probabilidad de mantenerse en el poder es $\bar{\gamma}^{\varsigma}$. Esta probabilidad es inferior a 1, por lo que existe la posibilidad de que el otro grupo le haga caer a pesar del apoyo del grupo del líder. Si los de su propio grupo dejan de apoyarlo, entonces cae automáticamente. En caso de que el líder caiga, la probabilidad de que el siguiente sea del mismo grupo es inferior a si el grupo apoya al actual.

En este contexto, Padró i Miquel elabora las funciones de utilidad de cada uno de los agentes implicados. Básicamente, los ciudadanos querrán maximizar su capacidad de consumo procedente del rendimiento de sus actividades económicas menos impuestos más gasto público del que puedan apropiarse. Por su parte, lo que quiere maximizar el líder es el dinero de los impuestos que puede desviar hacia su propio patrimonio. La utilidad de un ciudadano del grupo $A$ en un estado $S$ es la siguiente:

$$
\begin{gathered}
C\left(S, z^{A}\right)=\left(1-z^{A}\right)\left(\omega^{A}-\tau^{S a}\right)+ \\
z^{A}\left(\omega^{A}-\theta^{A}-\tau^{S b}+R\left(\eta^{A}\right)\right.
\end{gathered}
$$

Donde el componente $z$ denota la proporción del grupo $A$ que se dedica a cada una de las dos actividades económicas posibles y $R$ es la función de utilidad del gasto público que favorece al grupo étnico $A$. Por lo tanto, la utilidad depende positivamente de las rentas recibidas de cada actividad económica, que dependen de la proporción de tiempo que se dedica a cada una de ellas, negativamente de los impuestos que gravan cada una de ellas, negativamente de las ineficiencias derivadas de dedicar tiempo a la actividad en la que se tiene una desventaja competitiva y positivamente del gasto público que favorece al grupo étnico del individuo. 
Crespo, Galiano, López-Barceló, Merchante, Navarro de Vicente-Gella y Montes Gan

Por su parte, cuando ejerce el poder, el líder del grupo $A$ tiene la siguiente función de utilidad:

$$
\begin{gathered}
U^{A}=\tau^{A a}\left(\Pi^{A}\left(1-z^{A}\right)+\left(1-\Pi^{A}\right) z^{B}\right)+\tau^{A b}\left(\Pi^{A} z^{A}\right. \\
\left.\quad+\left(1-\Pi^{A}\right)\left(1-z^{B}\right)\right)-\Pi^{A} \eta^{A A}-\left(1-\Pi^{A}\right) \eta^{A B}
\end{gathered}
$$

Por lo tanto, la utilidad depende positivamente de la recaudación que el líder del grupo A pueda obtener de gravar cada actividad económica ejercida por cada grupo étnico, menos el gasto público dirigido a cada uno de los dos grupos étnicos. En caso de que el líder no esté en el poder, su utilidad será cero.

En estas circunstancias, la primera consecuencia evidente es que el líder no realizará gasto público que favorezca al otro grupo étnico y gravará la actividad económica en la que tienen ventaja comparativa lo máximo posible, siempre que ese impuesto se mantenga dentro de un nivel en el que no les compense cambiar de actividad. Los individuos del grupo del líder ( $A$, en el ejemplo) serán entonces conscientes de que, si el poder cambia de manos y pasa a un líder del grupo $B$, éste les tratará de la misma manera que el líder del grupo $A$ está tratando al grupo étnico $B$. Esto generará unos incentivos, por parte de los individuos del grupo $A$, a apoyar a su líder tales que estarán dispuestos a aceptar que éste les grave por las actividades en las que tienen ventaja comparativa y que el líder les pueda robar también a ellos. Esto es así porque, si tratan de disciplinar a su líder, correrán un mayor riesgo de acabar con un líder del otro grupo, que les dejará en una situación peor. Por ello, toleran cierto nivel de abusos de su líder. Por otro lado, para los individuos del grupo $A$, lo relevante será la diferencia (negativa) entre los gastos públicos dirigidos a ellos que reciben y los impuestos que tienen que pagar. En este contexto, el modelo también puede explicar un nivel elevado de gasto público para el desarrollo de redes clientelares entre los individuos del grupo $A$, ya que esto permite elevar los impuestos sobre la actividad económica en la que el grupo $A$ tiene ventaja comparativa sin por ello perder el apoyo de este grupo, pero también permite incrementar más los impuestos sobre la otra actividad económica y, como no realiza ningún gasto que favorezca al grupo $B$, esta mayor recaudación sobre la actividad $b$ permite directamente un mayor enriquecimiento personal del líder perteneciente al grupo $A$. Al mismo tiempo, cuanto mayor sean las fricciones o rigideces en la economía —el parámetro $\theta$-, también será mayor el grado de aprovechamiento al que el líder puede someter a los ciudadanos.

Esta situación puede explicar el enriquecimiento de muchos líderes no democráticos del África Subsahariana que, a pesar de su corrupción, siguen siendo apoyados por su grupo étnico, aunque a ellos también les están robando. Sin embargo, el despliegue de redes clientelares $\left(\eta^{A A}\right)$ y, sobre todo, el miedo a la llegada de los rivales explica que mantengan a su líder a pesar de los impuestos y sobornos que les obliga a pagar, y de su deficiente rendición de cuentas. Cuanto mayor sea el miedo que el líder sea capaz de inspirar entre los suyos, respecto a una hipotética llegada de los otros, mayor será su capacidad de robarles. Esto pronostica una política del miedo a los otros, a los distintos, como manera de lograr en mayor medida fines espurios de la clase dirigente.

$Y$ es aquí donde el modelo resulta tener una aplicación mucho más amplia que la inicialmente prevista —África Subsahariana-, ya que estos discursos del miedo se pueden constatar continuamente por todo el mundo, incluidas las democracias desarrolladas. En España, tanto a nivel nacional como a nivel de $\triangleright$ 
muchas comunidades autónomas, se constata una confrontación política que parece tener por objetivo maximizar el miedo de los votantes al ejercicio del poder que podrían ejercer los contrarios (o exagerar, por parte de la oposición, las maldades en el ejercicio del poder por parte del Gobierno de turno). El modelo de Padró i Miquel es revelador a la hora de entender que puede tratarse de una maniobra para aumentar la tolerancia, por parte de los votantes de un partido, a la corrupción de los políticos del mismo y para una menor exigencia de rendición de cuentas.

Finalmente, el profesor Padró i Miquel expuso un ejercicio de contrastación realizado en 2015 (Burgess et al., 2015) en el que se podía constatar, con la experiencia de Kenia, cómo la política de infraestructuras de carreteras desde la independencia del país había favorecido claramente al grupo étnico del líder nacional y cómo el gasto y el incremento en kilómetros de carreteras que favorecía a ese grupo étnico caía sustancialmente cuando el liderazgo político pasaba a otro grupo étnico. También se constataba que este sesgo era claramente mayor en los periodos dictatoriales que en los democráticos.

\subsection{Good Economics for Hard Times. Esther Duflo, MIT}

La economista, Esther Duflo, Premio Nobel de Economía en 2019 y Premio Princesa de Asturias en Ciencias Sociales en 2015, cerró el «Workshop in International Economics 2019» con una ponencia en la que anticipó las líneas principales de su nuevo libro, Good Economics for Hard Times, escrito junto al profesor Abhijit V. Banerjee, publicado en noviembre de 2019.
Por sí mismo, el título es un alegato en favor de la economía como ciencia social, y de la «buena economía» no solo como necesaria, sino como imprescindible ante los desafíos a los que nos enfrentamos hoy día, tales como el brexit, el auge de los populismos, los fenómenos migratorios, el impacto de la globalización y la apertura al comercio internacional, la sostenibilidad del crecimiento económico, la reducción de la desigualdad o la oportunidad para repensar las políticas sociales.

El argumento en el que se basa Duflo parte de la existencia de una visión muy crítica de la economía y de los economistas por parte de la sociedad. Esta crítica generalizada, agudizada tras la crisis, se apoya en su falta de respuesta adecuada a los grandes desafíos planteados en la actualidad, habiendo sido incapaces de anticipar y prevenir los efectos de situaciones a las que nos venimos enfrentando en las dos últimas décadas. En la práctica, esta pérdida de confianza se ha traducido en una búsqueda de soluciones desde un punto de vista personal, emocional y, a menudo, ideológico sin un fundamento económico sólido. Esa es precisamente la tarea que tienen frente a sí los economistas, sin renunciar a ofrecer respuestas desde la racionalidad económica a los problemas de hoy, conscientes de la complejidad del mundo, la falta de conocimiento sobre cómo funcionan las relaciones económicas y la existencia de opiniones contrapuestas. De hecho, este es el principal desafío: dar una respuesta a los problemas económicos críticos de nuestra sociedad.

Con este punto de partida, la profesora Duflo planteó su ponencia sobre cómo, desde la perspectiva de la economía del desarrollo y sobre una base científica y experimental sólida, se puede profundizar en el conocimiento de los problemas actuales que afectan a las $D$ 
personas a nivel mundial, en los países más y menos avanzados, ricos y pobres, y ofrecer respuestas desde la ciencia económica que sirvan de guía para la toma de decisiones. El análisis microeconómico de estas situaciones y las medidas adoptadas para abordarlas constituyen el eje central del trabajo, enfrentando la concepción tradicional por la que los individuos guían sus decisiones en la existencia de incentivos económicos con una nueva perspectiva en la que las decisiones obedecen a cuestiones de identidad -concepción y objetivos de uno mismo-. El análisis del comportamiento de las personas es determinante para guiar las decisiones de política económica y lograr cambios.

Para exponerlo de forma sencilla, la ponencia se centró en dos de las cuestiones analizadas en el libro: los flujos migratorios y la apertura comercial, con el objetivo no de ofrecer un análisis completo de los problemas, sino de poner el foco en los efectos personales y sociales que las medidas adoptadas — siguiendo la lógica económica - generan, y ofrecer algunas respuestas que puedan influir en las decisiones de política económica.

En relación con los flujos migratorios, la profesora Duflo señala cómo la visión existente al respecto, tanto por parte de la sociedad como de los economistas, ha guiado las decisiones, considerando que, si bien las migraciones tienen efectos positivos, también surgen riesgos en términos de caída de salarios y variación del empleo ${ }^{4}$. Este análisis, sin embargo, se puede enriquecer al considerar otros elementos que pueden influir en la decisión de migrar -y que podrían requerir otro tipo de políticas-, e incluso cabría preguntarse si el

\footnotetext{
4 En el caso de los trabajadores más cualificados hay una competencia real por el empleo, pero en el de los trabajadores menos cualificados no se compite por los mismos empleos, al aceptar, por lo general, los inmigrantes peores empleos, peores condiciones de trabajo y salarios más bajos.
}

diferencial de salarios entre nacionales e inmigrantes influye en los nuevos flujos migratorios, o lo que es más, cuáles serían las motivaciones que están detrás de la decisión de migrar. A priori, la expectativa de mejores oportunidades de vida y una trayectoria laboral más exitosa serían motivos suficientes, pero también es necesario considerar otros elementos que actúan como restricciones a la movilidad: la incertidumbre sobre el éxito de la migración, ya que los mercados de migrantes son delgados, los migrantes compiten por los mismos empleos y la experiencia puede resultar un fracaso personal (miedo a enfrentarse a sí mismos), o la falta de información certera (opiniones distorsionadas sobre los costes y beneficios de las migraciones), que lleva a tener expectativas sobre salarios y empleos mayores a los reales. Esto permite concluir que es precisamente la existencia o no de barreras a la movilidad la que guía estos flujos migratorios y que su reducción, en su caso, debería ser el objetivo que orientase las políticas adoptadas.

Una conclusión similar se plantea en relación a las políticas de apertura comercial. La teoría del comercio internacional basada en la teoría de la ventaja comparativa señala que cada país se especializa en aquello en que es mejor relativamente respecto a otro país y el ajuste se produce por la movilidad de factores entre países y sectores, provocando una igualación de las remuneraciones a los factores de producción.

Dado que en la práctica esta teoría no se cumple, la apertura comercial termina provocando mayor desigualdad. La profesora Duflo se apoya en la evidencia empírica existente en muchos países de Latinoamérica y, especialmente, en los trabajos de Topalova (2005) sobre el efecto de una reducción de aranceles en India en 1991 y sobre China realizado por Autor et al. (2016) para determinar que el $\triangleright$ 
elemento que está detrás de la falta de ajuste es la existencia de barreras a la movilidad del factor trabajo. Para el caso de India, además, la reputación es decisiva para tomar una posición ventajosa en el comercio internacional.

A este respecto, se concluye que las rigideces en la movilidad de los factores productivos, principalmente trabajo, pero también capital, tienen un papel decisivo en la adopción de políticas económicas generalistas, que no han resultado beneficiosas para la sociedad, y han generado mayor desigualdad y aumento de las brechas regionales y sociales. La opción sería apostar por políticas económicas dirigidas a los territorios o colectivos específicos que han asumido los costes más negativos de las profundas transformaciones vividas en los años más recientes; en los países más avanzados con medidas dirigidas a colectivos más perjudicados, como los trabajadores de mayor edad o los jóvenes, y en los países menos avanzados con políticas que favorezcan la movilidad (de bienes, capital, factores) y otras que podrían generar mayor desarrollo y bienestar para la sociedad: transporte, viviendas, educación y protección social, entre otras.

En todo caso, la intervención de la profesora Duflo concluyó que no existe una combinación de políticas económicas que, con carácter general, impulsen el crecimiento, ni siquiera aquellas defendidas por la teoría del crecimiento endógeno. El nivel de prosperidad y crecimiento económico dependen cada vez más de cómo se asignan los recursos existentes, y ello parte de considerar que los individuos no se guían únicamente por incentivos económicos, sino también por la concepción de sí mismos y de la sociedad de la que forman parte, y esto deber incluirse en las decisiones de política económica, prestando una mayor atención a los aspectos sociales.

\section{Conclusiones}

En definitiva, la edición de 2019 del WIE ha supuesto, para los asistentes, una privilegiada oportunidad de aproximarse al horizonte actual de la investigación en materia de economía internacional. La amplia variedad de temas presentados y de enfoques adoptados revela no solo la vitalidad de esta disciplina, sino el interés del debate actual sobre economía internacional y los retos y oportunidades que la economía de los datos ofrece para su análisis.

La orientación hacia una mayor intervención del Estado para compensar a los colectivos perdedores en la liberalización comercial, y la oportunidad que ofrecen las nuevas técnicas de tratamiento de datos para el análisis económico son las principales lecciones extraídas del evento.

\section{Bibliografía}

Autor, D. H., Dorn, D., y Hanson, G. H. (2016). The China Shock: Learning from Labor-Market Adjustment to Large Changes in Trade. Annual Review of Economics, 8, 205-240.

Basu, S. (2019). Are Price-Cost Markups Rising in the United States? A Discussion of the Evidence. Journal of Economic Perspectives, 33(3), 3-22.

Baumrind, D. (1967). Child Care Practices Anteceding Three Patterns of Preschool Behavior. Genetic Psychology Monographs, 75(1), 43-88.

Burgess, R., Jedwab, R., Miguel, E., Morjaria, A., y Padró i Miquel, G. (2015). The Value of Democracy: Evidence from Road Building in Kenya. American Economic Review, 105(6), 1817-51. Recuperado de https://pubs.aeaweb.org/doi/ pdfplus/10.1257/aer.20131031 
Crespo, Galiano, López-Barceló, Merchante, Navarro de Vicente-Gella y Montes Gan

Doepke, M., y Zilibotti, F. (2017). Parenting with Style: Altruism and Paternalism in Intergenerational Preference Transmission. Econometrica, 85(5), 1331-1371.

Doepke, M., Sorrenti, G., y Zilibotti, F. (2019). The Economics of Parenting. Annual Review of Economics 2019, 11(1), 55-84.

Doepke, M., y Zilibotti, F. (2019). Love, money, and parenting: how economics explains the way we raise our kids. Princeton University Press. doi:10.2307/j.ctvc77fr1

Easterly, W., y Levine, R. (1997). Africa's Growth Tragedy: Policies and Ethnic Divisions. The Quarterly Journal of Economics, 112(4), 1203-1250. Recuperado de https://inequality.stanford.edu/sites/ default/files/media/_media/pdf/Classic_Media/ Easterley\%20and\%20Levine_1997_Development\%20Economics.pdf

Gennaioli, N., y Shleifer, A. (2019). A Crisis of Beliefs: Investor Psychology and Financial Fragility. Princeton University Press.

Greenwood, R., y Hanson, S. (2013). Issuer Quality and Corporate Bond Returns. Review of Financial Studies, 26(6), 1483-1525.
Greenwood, R., y Shleifer, A. (2014). Expectations of Returns and Expected Returns. Review of Financial Studies, 27(3), 714-746.

Hausmann, R., y Sturzenegger, F. (2006). Global Imbalances or Bad Accounting? The Missing Dark Matter in the Wealth of Nations. CID Working Paper, (124).

Lang, V. F., y Tavares, M. M. (2018). The Distribution of Gains from Globalization, International Monetary Fund. Working Paper, (18/54).

Padró i Miquel, G. (2007). The Control of Politicians in Divided Societies: The Politics of Fear. Review of Economic Studies 74(4), 1259-1274. Recuperado de https://www.jstor.org/stable/4626180? seq=1\#page_scan_tab_contents

Rodrik, D. (1997). Has globalization gone too far? Challenge, 41(2), 81-94.

Rodrik, D. (2011). The Globalization Paradox: Democracy and the Future of the World Economy. WW Norton \& Company.

Topalova, P. (2005). Trade liberalization, poverty and inequality: evidence from Indian districts. NBER Working Paper, (11614). 